\title{
FINE STRUCTURE LOCALIZATIONS OF ACID PHOSPHATASE IN RABBIT AND BULL SPERM HEADS
}

\author{
R. J. TEICHMAN* AND M. H. BERNSTEIN \\ Department of Anatomy, Wayne State University, \\ School of Medicine, Detroit, Michigan, U.S.A.
}

(Received 24th November 1970)

\begin{abstract}
Summary. In rabbit spermatozoa, acid phosphatase activity is primarily localized within unique, morphologically distinct bands beneath the equatorial segment of the acrosome; secondary localizations appear along the entire postacrosomal segment of the sperm head. In bull spermatozoa, acid phosphatase activity is associated with the anterior third of the postacrosomal segment. The enzyme was extractable with detergent after a pretreatment with pyrimidinetrione (barbituric acid). Activity was inhibited by sodium fluoride and destroyed by fixation. The localization of acid phosphatase in the postacrosomal segment of both species and the unique localizations in the rabbit equatorial segment may ultimately relate to sperm penetration or gamete membrane fusion mechanisms active during the fertilization process.
\end{abstract}

\section{INTRODUCTION}

Recent reports from a number of laboratories (Buongiorno-Nardelli \& Bertolini, 1967; Teichman \& Bernstein, 1969) have suggested that the sperm acrosome may be identified as a lysosome-like structure. Acid phosphatase is generally accepted as a lysosomal enzyme (DeDuve, 1963) and is the most widely used histochemical marker for the demonstration of lysosomes.

Histochemical electron microscopic studies of acid phosphatase distribution have shown significant and apparently species-specific localizations of the enzyme in the coverings of the sperm head. The data reported here are equivocal with regard to the lysosomal nature of the acrosome.

\section{MATERIALS AND METHODS}

Bull semen samples, obtained from the Michigan Animal Breeders Cooperative, East Lansing, Michigan, were processed individually while rabbit semen was pooled to a volume of at least $1.0 \mathrm{ml}$. Only fresh material was used in this study. Semen samples were diluted in $20.0 \mathrm{ml}$ of cold $0.25 \mathrm{M}$-sucrose, gently stirred and centrifuged at $500 \mathrm{~g}$ for $10 \mathrm{~min}$. The washing procedure was

\footnotetext{
* Present address: Department of Anatomy, School of Medicine, University of Hawaii.
} 
repeated once for rabbit and twice for bull semen. (All procedures were carried out at $4^{\circ} \mathrm{C}$.) Controls and samples needed for study of normal morphology were fixed in picric acid paraformaldehyde (Stefanini, Demartino \& Zamboni, 1967) after a sucrose wash.

\section{Histochemical studies}

Measurements were carried out in vitro with sucrose-washed spermatozoa. The results indicated a membrane-bound acid phosphatase activity. Solubilization was achieved by detergent extraction $(0 \cdot 1 \%$ Triton $\mathrm{X}-100)$ following pretreatment with 0.009 M-pyrimidinetrione (Teichman \& Bernstein, 1969) and acid phosphatase activity was determined spectrophotometrically (Straus, 1956) in acetate buffer, $\mathrm{pH} 5 \cdot 2$, with phenolphthaleinphosphate as the substrate.

Electron microscopic localization was achieved by the method of Gomori (1952) using sodium $\beta$-glycerophosphate as substrate and an incubation of 30 min at $37^{\circ} \mathrm{C}$. After all the incubations for enzyme localization, spermatozoa were fixed in picric acid paraformaldehyde, and post-fixed in $1 \%$ osmium tetroxide. Preliminary studies had shown that even neutral calcium formol fixation resulted in drastic reduction of acid phosphatase activity. Incubations for localization of the enzyme were, therefore, carried out before fixation.

Thin sections were stained with uranium and lead salts and examined in an RCA EMU-3E electron microscope. No lead stains were used on any of the sections for enzyme localizations.

\section{RESULTS}

The heads of rabbit and bull spermatozoa are similar in general proportions (Pl. 1, Fig. 1 and Pl. 4, Fig. 7) although those of the bull spermatozoa are considerably longer and slightly thinner. The acrosome is membrane-limited throughout and its membranes are in closest approximation in the equatorial segment. Beneath the equatorial segment of the rabbit acrosome, bands are evident as membrane-limited subacrosomal structures containing a finely granular material (Pl. 1, Fig. 2).

Acid phosphatase activity is readily demonstrable in seminal plasma. In seven samples of bull semen, the plasma showed 0.73 units of activity, while thirteen samples of rabbit seminal plasma averaged 0.04 units (activity units $=$ $\mu \mathrm{g}$ of substrate liberated $/ \mu \mathrm{g}$ protein $/ \mathrm{hr}$ ). Sucrose-washed whole spermatozoa do not release acid phosphatase activity into the medium. Spermatozoa treated with pyrimidinetrione and detergent release almost all of their acid phosphatase activity. In such extracts of washed spermatozoa, $3 \cdot 2$ units of activity were obtained from bull spermatozoa and an average of 0.47 units from rabbit spermatozoa.

In rabbit spermatozoa, the most pronounced localization is in the equatorial bands. Reconstruction from a large number of sections shows the equatorial bands and the corresponding acid phosphatase localizations (Text-fig. 1) to curve around the sperm head in the equatorial segment. The arms of each band are displaced in the longitudinal axis and do not cross the midline. Sections are shown (Pl. 2, Fig. 3) taken in planes corresponding to A to $\mathrm{H}$ in 
Text-fig. 1. As suggested by the model, Sections D, B, H and F show one, two, three and four equatorial localizations.

Secondary localizations of acid phosphatase activity in the heads of rabbit spermatozoa are seen in the subacrosomal areas (Pl. 2, Fig. 3, Sections A, C, D, $\mathbf{E}$ and $\mathrm{H})$; this activity is continuous into the postacrosomal area (Pl. 2, Fig. 3, Sections D, B and E) where the activity becomes more superficial or, at least, further separated from the nucleus and is associated with the postacrosomal dense lamina (Pl. 2, Section G).

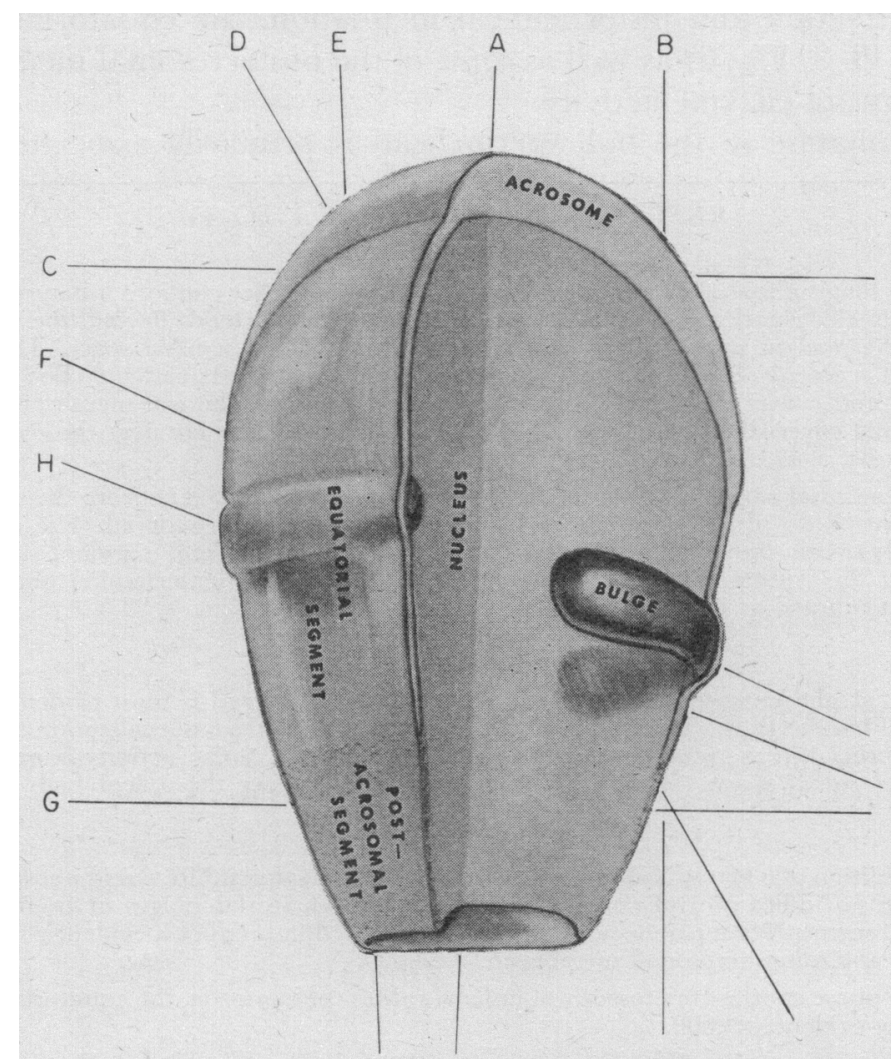

TEXT-FIG. I. A reconstruction of the rabbit sperm head and the equatorial bands. The two bands (bulges) are limited to and curve around the sperm head at the anterior and posterior limits of the equatorial segment. The arms of each band are displaced in the longitudinal axis and do not extend to the midline of the head.

Further sites of acid phosphatase activity can be seen around the mitochondria of the middle piece and in the axial components of the flagellum (Pl. 2, Sections A and E).

The specificity and validity of the localizations were established in control studies which demonstrated that there were no localizations in the absence of added substrate. The addition of $0.005 \mathrm{M}-\mathrm{NaF}$ completely inhibited the activity in the head membranes, although some endogenous activity can still be seen in the outer acrosomal membrane (Pl. 3, Fig. 4). 
The persistent appearance of the equatorial bands led us to re-examine the rabbit spermatozoon by the light microscope. A phase-contrast photomicrograph (PI. 3, Fig. 5) gives a clear indication of the presence of the equatorial bands.

Biochemical studies have indicated that the acid phosphatase activity of the rabbit spermatozoon is membrane-bound and can be released by detergent treatment. A sample of rabbit spermatozoa extracted with Triton X-100 is seen in Pl. 3, Fig. 6. At least $80 \%$ of the total activity has been removed from the heads and the bulk of the remaining activity is in the flagellum. Even after detergent extraction and histochemical incubation, the equatorial bands are still present (Pl. 3, Fig. 6) as well as some of the postacrosomal membranes but almost no signs of enzyme activity.

Acid phosphatase in the bull sperm head is primarily associated with the

\section{EXPLANATION OF PLATES 1 TO 4}

PLATE 1

Fig. 1. In longitudinal section, the rabbit sperm acrosome (Ac) contains a homogeneous moderately electron-dense material. An apical body (ApB) extends beyond the anterior limit of the nucleus under the inner acrosome membrane as a filled space. The postacrosomal segment (PAc) extends from the thinned equatorial segment (EqS) of the acrosome and covers the posterior third of the sperm head. The cell membrane (Cm) is intact and covers the entire head, with tightest adherence evident over the equatorial bands $(\mathrm{EqB}) . \times 44,000$.

Frg. 2. Equatorial bands $(\mathrm{EqB})$ in the rabbit sperm head are located beneath the equatorial segment (EqS) of the acrosome and contain a fine granular material. The junction (arrow) between the equatorial segment and the postacrosomal segment (PAc) is evident at the bottom of this figure. Note the postacrosomal dense lamina underlying the cell membrane as a component of the postacrosomal segment. $\times 88,000$.

PLATE 2

FIG. 3. Acid phosphatase localization in the rabbit sperm head is most evident in the equatorial bands (EqB) (Sections B, D, E, F and H). The enzyme localization becomes more superficial in the postacrosomal segment (Section $G$ ). Some activity is present in the subacrosomal space (Section $\mathrm{C}$ ) and is continuous over the apical body (ApB). $\times 23,000$.

PLATE 3

FIg. 4. Addition of $0.005 \mathrm{M}-\mathrm{NaF}$ to washed rabbit spermatozoa inhibits acid phosphatase. There are no indications of enzyme activity in the equatorial bulges or in the postacrosomal segment. Some non-specific endogenous reaction product is evident within the flagellum and outer acrosomal membrane. $\times 23,000$.

FIG. 5. In phase contrast microscopy of unfixed rabbit spermatozoa, the equatorial bands are clearly evident. $\times 2000$.

Fig. 6. Extraction of rabbit spermatozoa with pyrimidinetrione and Triton X-100 results in removal of almost all activity from the persistent equatorial bands (arrows) and postacrosomal segment. $\times 23,000$.

\section{PLATE 4}

FIG. 7. In longitudinal section, the bull sperm head reveals a smooth regular surface. The dense nucleus exhibits an anterior taper with the acrosome (Ac) closely adherent to its anterior half. An apical body $(\mathrm{ApB})$ is present within the anterior enlargement of the acrosome as well as a single apical density (AD). The thinned equatorial segment (EqS) of the acrosome is evident and the loose postacrosomal segment (PAc) covers the posterior half of the sperm nucleus. $\times 23,000$.

Fig. 8. A section across the postacrosomal segment (broken line, Fig. 9) of the head of a bull spermatozoon shows that acid phosphatase activity is continuous around the head and is limited to the outer aspect of the postacrosomal segment. $\times 23,000$.

FIG. 9. A longitudinal section of a bull spermatozoon that has been incubated to show acid phosphatase activity. The localization is most prominent in the anterior third of the postacrosomal segment (PAc). Mitochondrial (Mi) and paraxial activity is also evident. The broken line indicates the plane of the section shown in Fig. 8. $\times 23,000$. 
PIATE I

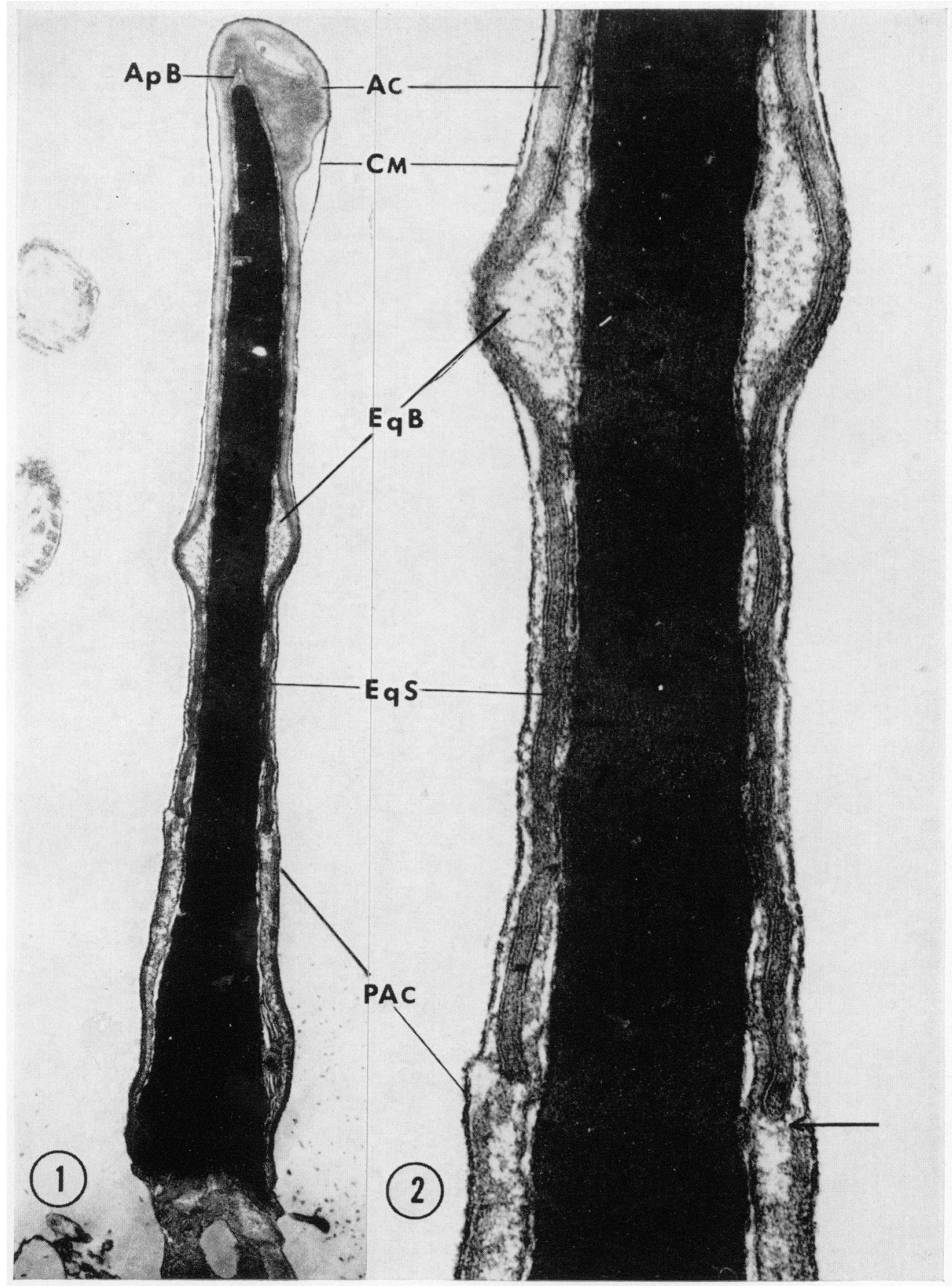

(Facing 1.246 ) 
PLATE 2

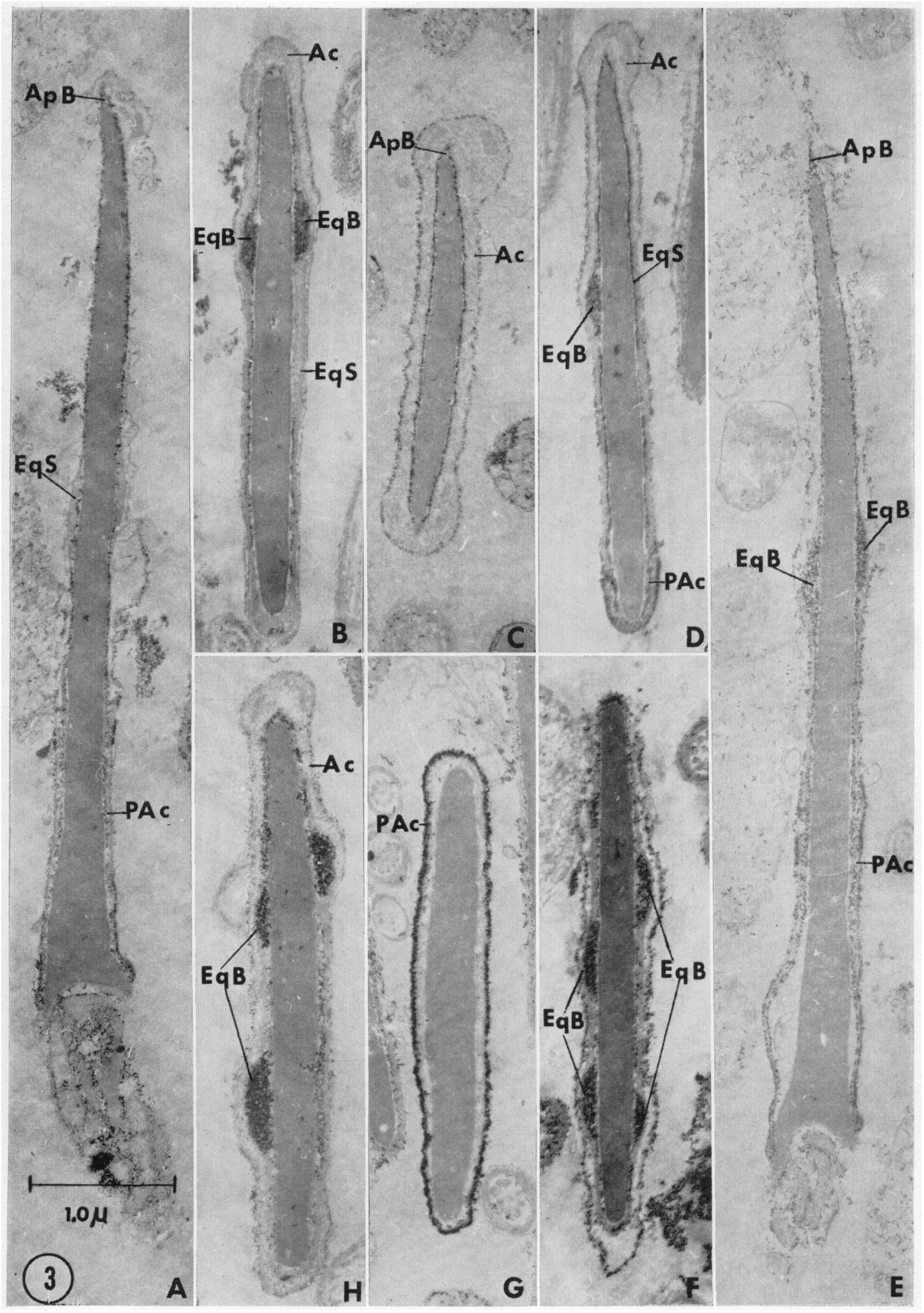


PLATTE 3
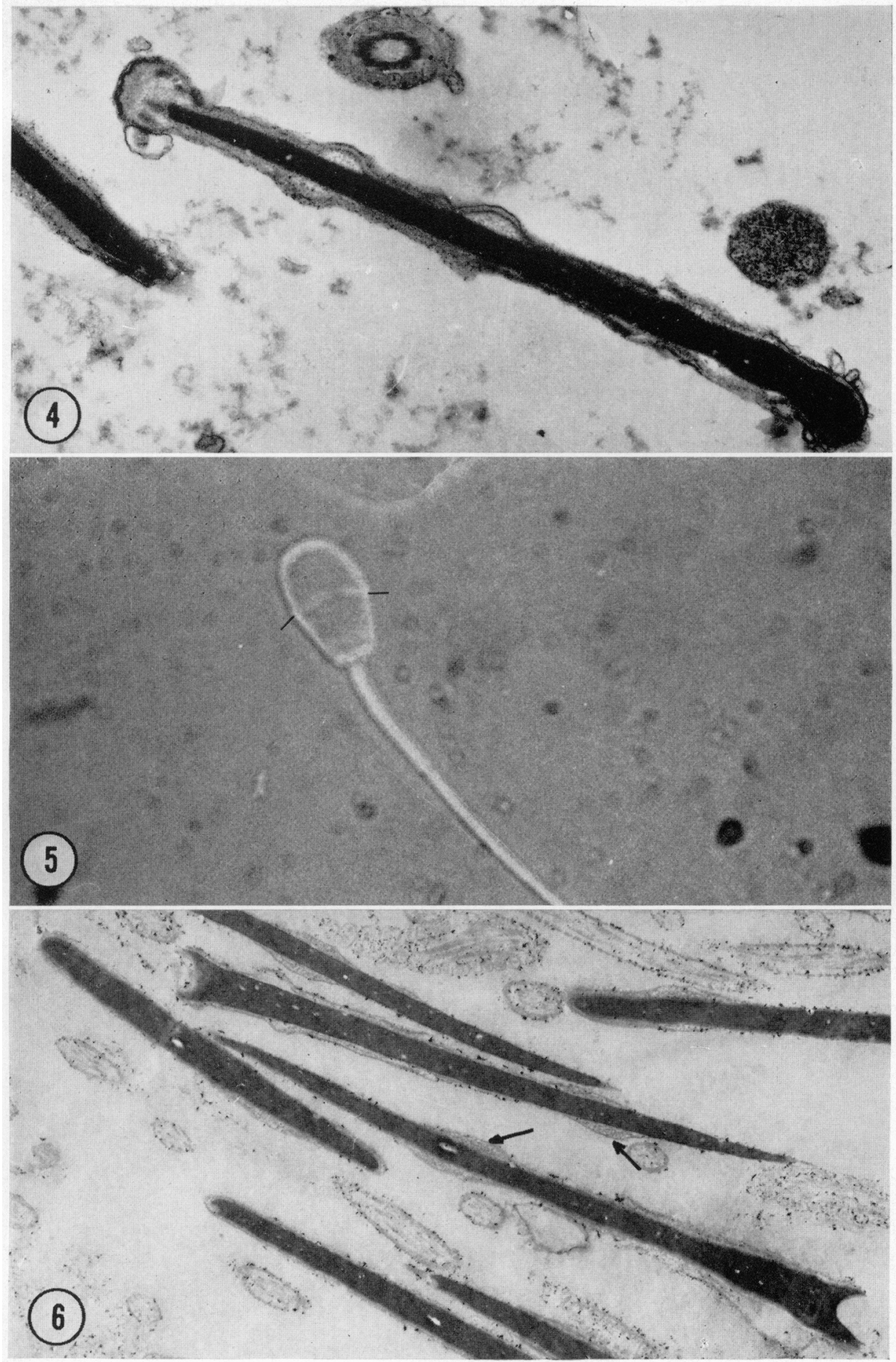
PLATE 4

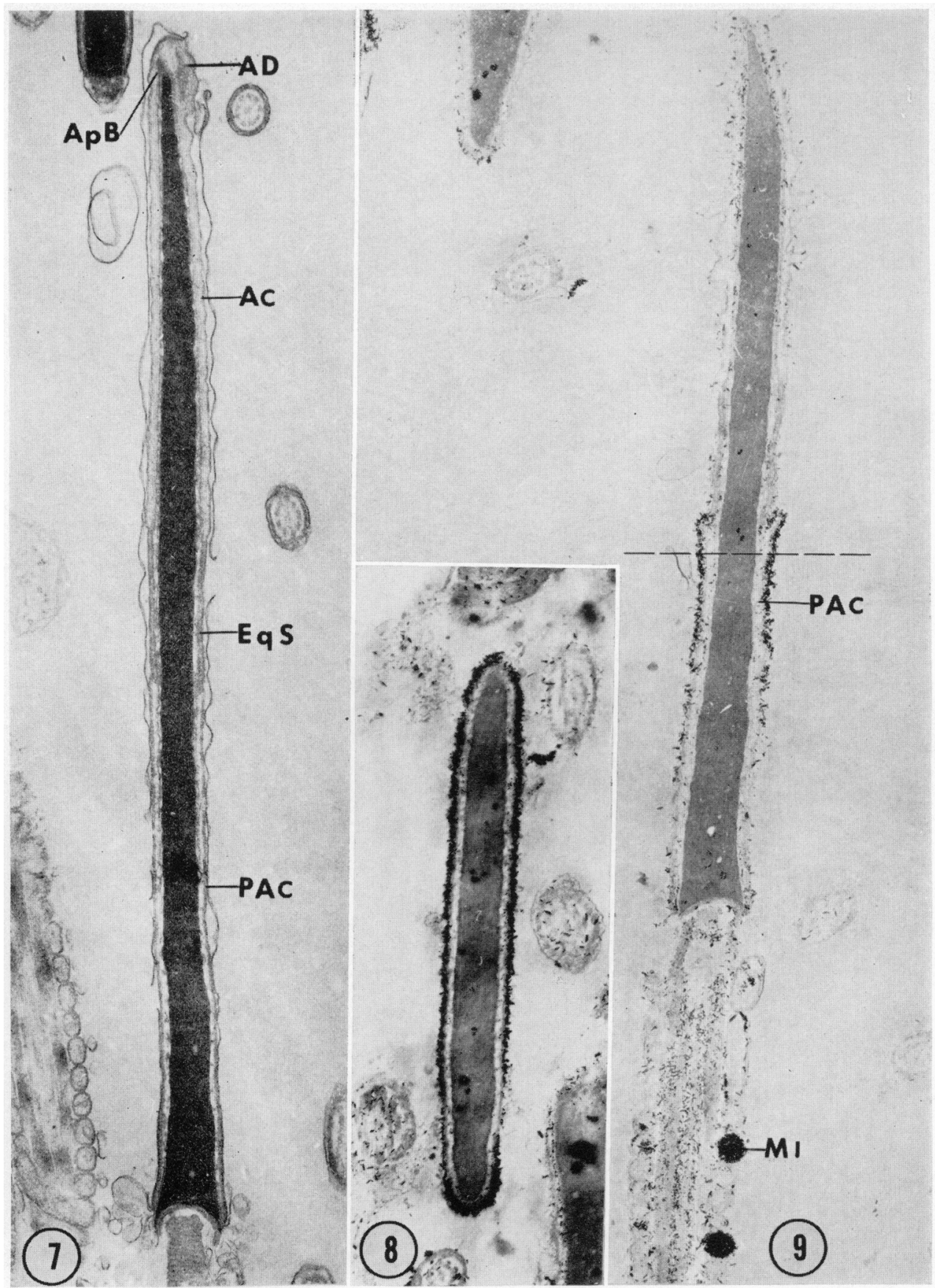


anterior third of the postacrosomal segment (Pl. 4, Fig. 9). There appear to be no equatorial bands present in the bull sperm head and only modest subacrosomal activity is present. A cross-section (Pl. 4, Fig. 8) of the anterior portion of the postacrosomal segment shows the enzyme activity has also become superficial to the subacrosomal or nuclear envelope localizations seen in more anterior areas. To recognize the similarities of the postacrosomal localizations in bull and rabbit spermatozoa, Pl. 4, Fig. 8 should be compared with Pl. 2, Sections $\mathrm{G}$ and $\mathrm{G}$.

\section{DISCUSSION}

This and much other work has been motivated by the acrosome-lysosome analogy. Buongiorno-Nardelli \& Bertolini (1967) have demonstrated the association of acid phosphatase and four other acid hydrolases with the acrosome of Triturus cristatus spermatozoa. Gordon \& Barnett (1967) have demonstrated neutral and alkaline ATP-ase activity in guinea-pig spermatozoa, primarily in mitochondrial and flagellar relations. The results of the present study indicate that the acid phosphatase of rabbit and bull sperm heads relates to the subequatorial and postacrosomal regions rather than to the acrosome.

Recent observations by Yanagimachi (1966), Yanagimachi \& Noda (1970), Szollosi \& Ris (1961) and Barros \& Franklin (1968) on the entry of spermatozoa into mammalian ova indicate that the equatorial and postacrosomal regions are the sites of initial contact. Fusion with the egg membrane is followed by midlateral (equatorial) entry of the sperm head into the egg cytoplasm. Koehler (1969) has suggested that the area of the equatorial segment-postacrosomal junction may encompass a species-specific structural feature of spermatozoa essential for recognition or attachment mechanisms during fertilization and subsequent egg activation. These data suggest that lysosomal enzymes essential for sperm penetration and membrane fusion may be differentially distributed in sperm head regions. These and other studies (Ericsson, 1969) increasingly suggest the importance of lysosomal activity in the events relating to fertilization. Stambaugh \& Buckley (1969) reported negative findings for acid phosphatase activity. However, the present report along with that of Allison \& Hartree (1970) suggest that difficulties or failure in demonstrations of acid phosphatase are probably due to an association of the enzyme with stable membrane components of the sperm heads.

\section{ACKNOWLEDGMENT}

This work was supported by Grant No. HD-03416-02 from the U.S.P.H.S. and constituted part of the dissertation of Robert J. Teichman submitted to the Graduate Division of Wayne State University in partial fulfilment of the requirements for the degree of Doctor of Philosophy.

\section{REFERENCES}

Allison, A. G. \& Hartree, E. F. (1970) Lysosomal enzymes in the acrosome and their possible rôle in fertilization. F. Reprod. Fert. 21, 501. 
BarRos, C. \& Frankin, L. E. (1968) Behavior of the gamete membranes during sperm entry into the mammalian egg. F. Cell Biol. 37, G13.

Buongrorno-NARdeli, M. \& Bertolini, B. (1967) Subcellular localization of some acid hydrolases in Triturus cristatus spermatozoa. Histochemie, 8, 34 .

DeDuve, C. (1963) The lysosome concept. In: Ciba Foundation Symposium on Lysosomes, pp. 1-31. Eds. A. V. S. DeReuck and M. P. Cameron. Little, Brown, Boston.

ERICsson, R. (1969) Capacitation in vitro of rabbit sperm with mule eosinophils. Nature, Lond. 221, 568.

GoMORI, G. (1952) Microscopic histochemistry: principles and practices, p. 193. University of Chicago Press.

Gordon, M. \& BARNETT, R. (1967) Fine structural cytochemical localizations of phosphatase activities of rat and guinea pig. Expl Cell Res. 48, 395.

KOEHLER, J. K. (1969) The acrosome-post-nuclear cap junction. A characteristic landmark in rabbit spermatozoa revealed by freeze-etching. 7. Cell Biol. 43, 70a.

Stambaugh, R. \& Buckrey, J. (1969) Identification and subcellular localization of the enzymes effecting penetration of the zona pellucida by rabbit spermatozoa. F. Reprod. Fert. 19, 423 .

Stefanini, M., Demartino, C. \& Zamboni, L. (1967) Fixation of ejaculated spermatozoa for electron microscopy. Nature, Lond. 216, 173.

STRAus, W. (1956) Concentration of acid phosphatase, ribonuclease, desoxyribonuclease, beta-glucuronidase and cathepsin in 'droplets' isolated from kidney cells of normal rats. 7. biophys. biochem. Cytol. 2, 513.

Szollosi, D. \& Ris, H. (1961) Observations on sperm penetration in the rat. F. biophys. biochem. Cytol. $10,275$.

Terchman, R. J. \& Bernstein, M. H. (1969) A cytochemical comparison of bull, human and rabbit acrosomes. F. Cell Biol. 43, 144a.

YanAGmaGhi, R. (1966) Time and process of sperm penetration into hamster ova in vivo and in vitro. 7. Reprod. Fert. 11, 359.

YANAGIMACHI, R. \& NoDA, Y. D. (1970) Electron microscope studies of sperm incorporation into the golden hamster egg. Am. F. Anat. 128, 429. 